\title{
Robust user identification based on facial action units unaffected by users' emotions
}

\author{
Ricardo Buettner \\ Aalen University, Germany \\ ricardo.buettner@hs-aalen.de
}

\begin{abstract}
We report on promising results concerning the identification of a user just based on its facial action units. The related Random Forests classifier which analyzed facial action unit activity captured by an ordinary webcam achieved very good values for accuracy (97.24 percent) and specificity (99.92 percent). In combination with a PIN request the degree of specificity raised to over 99.999 percent. The proposed biometrical method is unaffected by a user's emotions, easy to use, cost efficient, non-invasive, and contact-free and can be used in human-machine interaction as well as in secure access control systems.
\end{abstract}

\section{Introduction}

Robust user identification is a precondition of modern human-computer interaction systems, in particular of autonomous robots, not only for issues of security but also for convenience [1,2].

While biometric user identification by fingerprints or pulse response is not contactless and photography-based approaches (iris, retinal, and face scans) can be tricked by previously captured images [2]-[4], we investigate the possibility of identifying a person using its specific facial expressions measured by the amount of facial action units. Since these facial action units are not only user-specific [5] but also emotion-specific [6] and emotions substantially affect a broad range of user behavior e.g. mouse trajectories [7], the particular difficulty is the development of a robust user identification method unaffected by a user's emotions.

That is why in this paper we evaluate the possibility of reliably identifying a person based on its facial action units and unaffected by its emotions.

The research question is: "Can we robustly identify a user based on its facial action units and unaffected against its emotions?"

In order to identify the correct user we made use of a Random Forests decision tree classifier. The classifier uses neurophysiological data from a controlled laboratory experiment in which we continuously recorded facial action units while evoking specific emotions by displaying 14 normative emotional stimuli from the International Affective Picture System (IAPS) [8].

As a result we achieved a balanced prediction accuracy of 97.2 percent just based on the facial expressions. When we combine the biometric capture with a PIN request, which is a rule in access validation systems [2,3], we reached a very good false positive rate of only 7.633284e-06 (over 99.999 percent specificity).

On the basis of our results we can offer some interesting theoretical insights, e.g. which facial action units are the most predictive for user identification such as the lid tightener (orbicularis oculi, pars palpebralis) and the upper lip raiser (levator labii superioris). In addition our work has practical implications as the proposed contactless user identification mechanism can be applied as

- a comfortable way to continuously recognize people who are present in human-computer interaction settings, and

- an additional authentication mechanism in PIN entry systems.

The most important findings from these analyses are:

1) It is possible to identify a person based on its facial action units alone with an accuracy of 97.2 percent.

2) This user identification mechanism is unaffected by the user's emotions.

3) Important for secure access control, the specificity (true negative rate) is 99.92 percent.

4) In combination with a PIN request the degree of specificity raised to over 99.999 percent.

5) The most important facial muscles for robust user identification are the orbicularis oculi (pars palpebralis), levator labii superioris, and orbicularis oris.

The paper is organized as follows: Next we present an overview of the research background on facial expressions and the facial action coding system before providing the research methodology, including experimental procedure, stimuli, sample characteristics, measurements, data preparation, and the Random Forests decision tree method. After that we present the machine learning results concerning the performance evaluation and analysis of important specific facial action units and related facial muscles. We then discuss the results and include theoretical and practical implications, before concluding with limitations and suggestions for future research.

URI: http://hdl.handle.net/10125/49923

ISBN: 978-0-9981331-1-9 


\section{Research background on facial expressions and the facial action coding system}

The identification of a human by another human through the analysis of the others' face and its facial expressions is part of a long evolution over several phylogenetic [9] and ontogenetic hominid evolution stages [10]. Facial expression analysis is a key concept of communication and social competence $[9,11]$.

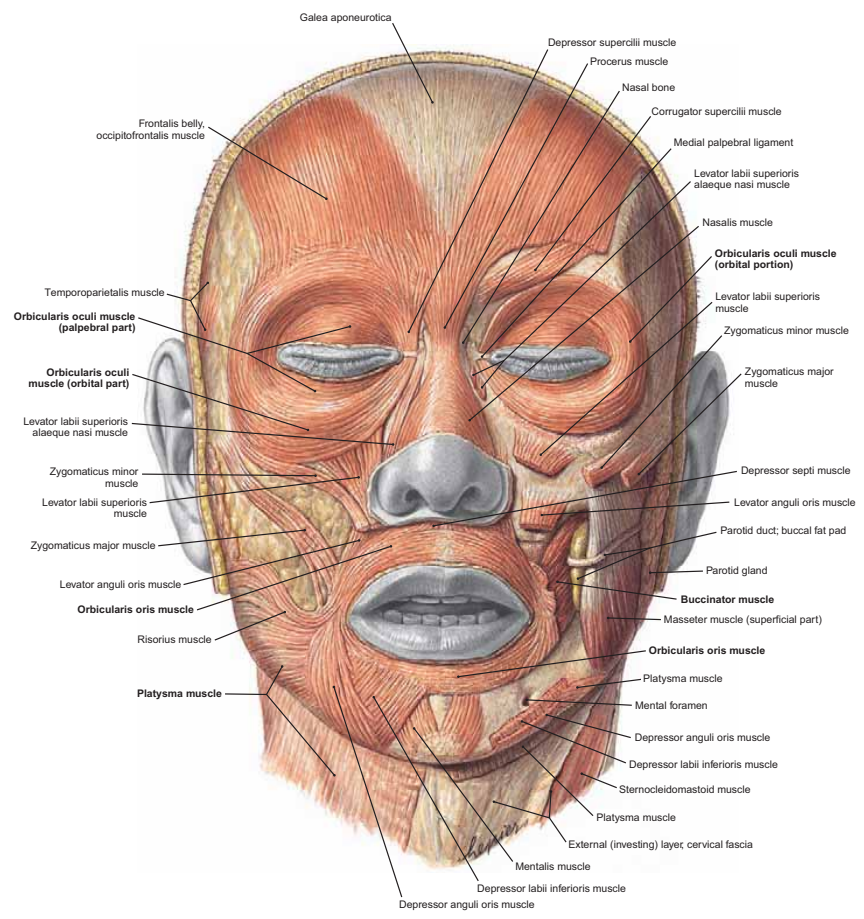

Figure 1: Muscles of facial expression (anterior view). From [12, p. 580].

Any human being's facial expression can be broken down into smaller facial actions, so-called facial action units, e.g. the raising of the inner brow (action unit 1) or outer brow (action unit 2). The facial action coding system (FACS) by Ekman et al. [6,13] is a system that describes all observable facial movements anatomically based on the contraction of specific facial muscles (see figure 1), e.g. zygomaticus major muscle for the lip corner puller (action unit 12).

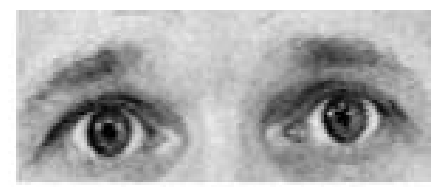

Figure 2: Activation of action unit 1: Inner portion of the brows is raised. From [14].

While it was found that the use of facial action units is partly unique for a user [5], it was also found that specific combinations of facial action units are related to specific

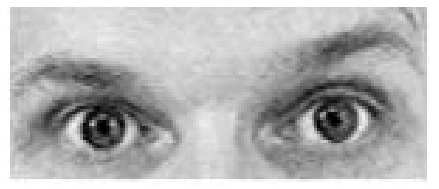

Figure 3: Activation of action unit 2: Outer portion of the brows is raised. From [14].

user emotions [15]. For instance, while happiness is related to the combination of action units 6 and 12 , contempt is related to action unit 14 [16].

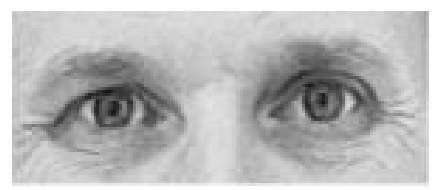

Figure 4: Cheeks are raised (action unit 6). From [14].

\section{Methodology}

In order to clearly contribute to NeuroIS research and show strong methodological rigor, we followed the NeuroIS guidelines provided by vom Brocke and Tiang [17]. To base the experimental design adequately on solid research in related fields of neuro-science we reviewed the fundamental anatomical mechanism of the relationship between specific facial expressions, their related facial action units and facial muscles $[6,13]$. The methodology uses camera-based facial action unit analysis as a well-established approach in physiology and psychology $[5,14,15]$. With this method, bio-data (i.e. facial action units related to specific facial muscles) can be used to better identify the correct user (cf. guideline 4 of [17]). In comparison to other neuroscience tools, camera-based facial action unit analysis is a contactfree and efficient method of choice. We further applied the guidelines and standards from the Noldus FaceReader 6 manual.

\subsection{Experimental procedure}

We chose a one group design (within-subject, proven emotional stimuli from the International Affective Picture System (IAPS) [8] as treatment (see table 1), completely randomized, double-blind, cf. [18]).

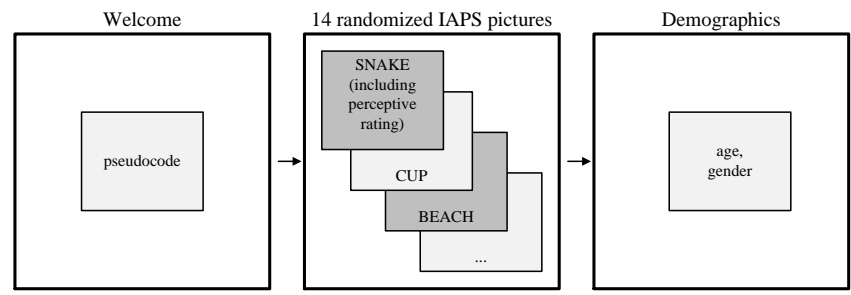

Figure 5: Test procedure. 
The standardized test procedure was as follows (figure 5). First, the laboratory assistant welcomed the participant and explained the laboratory environment in general. After that the participant was provided with information about the experiment and read this before signing a consent form. In the next step the fourteen IAPS stimuli (see table 1) were randomly presented on a computer and the participant had to evaluate its subjective perception of emotions that occurred by rating every stimulus relating to the seven basic human emotions (anger, contempt, disgust, happiness, sadness, fear, surprise) using a six-point Likert scale without a time limit. Finally, the participant had to answer demographic questions (age, gender). After completing the procedure we talked to the participant about the stimuli in order to ensure that they were in a good mental state before we discharged her or him.

\subsection{Stimuli}

To evoke specific emotions we used 14 pictures from the International Affective Picture System (IAPS). IAPS is a database designed for experimental investigations providing a standardized, emotionally-evocative, internationally accessible set of color pictures for studying emotion and attention [8]. We chose six pleasant, six unpleasant and two neutral pictures from IAPS (see table 1) and presented them completely at random to the participants.

\begin{tabular}{lll}
\hline No. & Name & Mood \\
\hline 1050 & Snake & negative \\
1201 & Spider & negative \\
1300 & Pit Bull & negative \\
2811 & Gun & negative \\
9001 & Cemetery & negative \\
9270 & Toxic Waste & negative \\
\hline 2030 & Woman & positive \\
2070 & Baby & positive \\
2306 & Boy & positive \\
2311 & Mother & positive \\
2341 & Children & positive \\
8540 & Athletes & positive \\
\hline 7001 & Buttons & neutral \\
7009 & Mug & neutral \\
\hline
\end{tabular}

Table 1: Specification of IAPS stimuli.

We controlled the subjective perception of emotions that occurred by asking the participants to rate every stimulus concerning the seven basic human emotions (anger, contempt, disgust, happiness, sadness, fear, surprise) using a six-point Likert scale. For example, we show the boxplots for the perceived participants' emotions in figures 6 (gun), 7 (buttons), and 8 (baby). All other perceived emotion ratings are shown in the appendix.

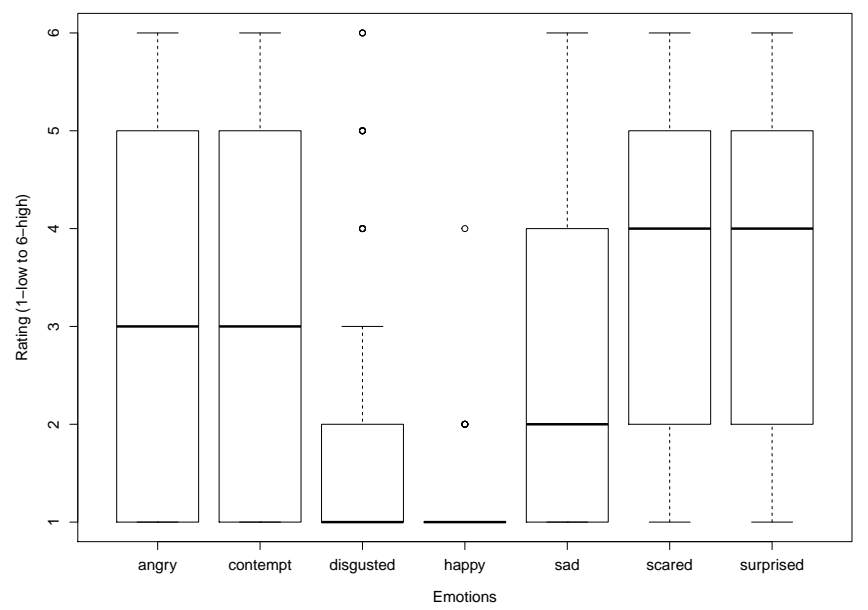

Figure 6: Perceptive rating of gun stimulus (\#2811).

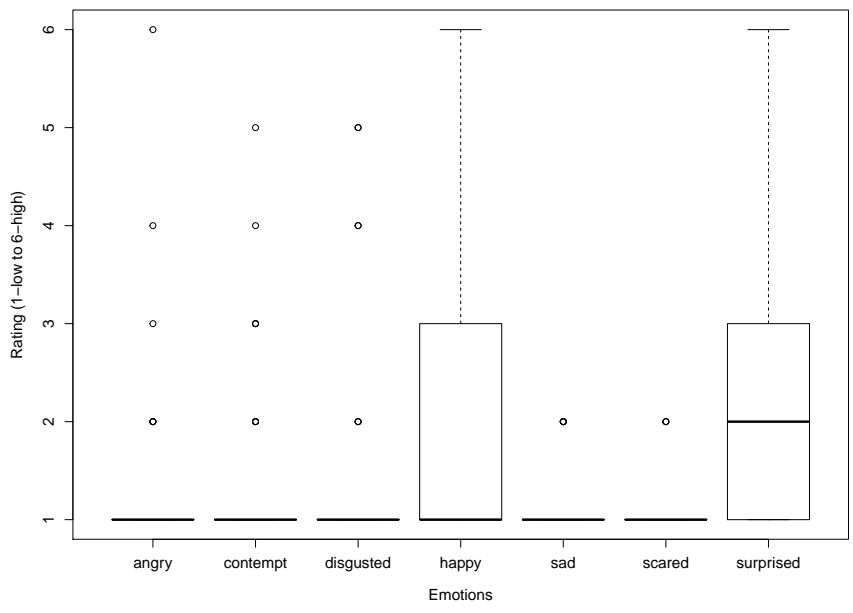

Figure 7: Perceptive rating of buttons stimulus (\#7001).

\subsection{Sample characteristics}

We recruited 106 participants to take part in the laboratory experiment. Two participants did not take part in the experiment after reading the declaration of consent and reporting that they had specific phobias (in one case arachnophobia, in the other case snake phobia). That is why we decided to exclude them from the experiment. In addition, data from two other participants tested in succession had to be removed due to technical reasons (time lag in loading stimuli due to a technical network IP conflict). The final dataset comprised 102 participants (48 females, 54 males) aged from 18 to 78 years $(M=41.3$, S.D.=15.2).

\subsection{Measurements}

Each of the twenty most common facial action units (see table 2) was measured using the Noldus FaceReader software analysing video material from an Axis M1054 IP camera (1280x720 pixel, 30 pictures per second). If action unit activity was detected, it was numerically coded in five 


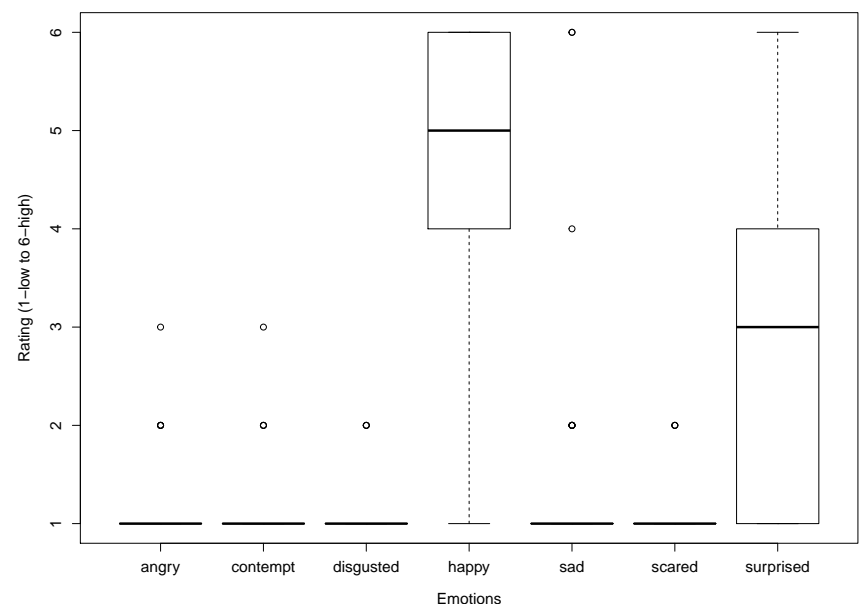

Figure 8: Perceptive rating of baby stimulus (\#2070).

intensities: 2 (trace), 4 (slight), 8 (pronounced), 16 (severe), 32 (max). In addition, it was measured when mouth, left eye, or right eye were open or closed and when the left or the right eyebrow were raised, neutral or lowered. This was also numerically coded.

\begin{tabular}{ll}
\hline AU Description & Related facial muscles \\
\hline \#01 Inner Brow Raiser & Frontalis, pars medialis \\
\#02 Outer Brow Raiser & Frontalis, pars lateralis \\
\#04 Brow Lowerer & Corrugator supercilii, Depressor supercilii \\
\#05 Upper Lid Raiser & Levator palpebrae superioris \\
\#06 Cheek Raiser & Orbicularis oculi, pars orbitalis \\
\#07 Lid Tightener & Orbicularis oculi, pars palpebralis \\
\#09 Nose Wrinkler & Levator labii superioris alaquae nasi \\
\#10 Upper Lip Raiser & Levator labii superioris \\
\#12 Lip Corner Puller & Zygomaticus major \\
\#14 Dimpler & Buccinator \\
\#15 Lip Corner Depres. Depressor anguli oris \\
\#17 Chin Raiser & Mentalis \\
\#18 Lip Puckerer & Incisivii labii superioris and Incisivii labii inferioris \\
\#20 Lip Stretcher & Risorius w/ platysma \\
\#23 Lip Tightener & Orbicularis oris \\
\#24 Lip Pressor & Orbicularis oris \\
\#25 Lips Part & Depressor labii inf. or relax. of Mentalis, or Orbic. oris \\
\#26 Jaw Drop & Masseter, relaxed Temporalis and internal Pterygoid \\
\#27 Mouth Stretch & Pterygoids, Digastric \\
\#43 Eyes Closed & Relax. of Levator palpebr. super.; Orbic. oculi, pars palpebr. \\
\end{tabular}

Table 2: Facial action units (AU) measured and related facial muscles $[19,20]$.

\subsection{Data preparation}

For all participants we recorded about 13 hours of facial expression material in total. For each of the 102 participants and for each of the 14 IAPS stimuli presented, we summarized the specific intensities of facial action unit activity, resulting in 1428 data records (102 times 14). For 15 of these records data were missed due to a temporally unfavourable head orientation by six of the participants. Thus, we had 1413 valid data records for further analysis.

Statistics (means and standard deviations) for all variables (facial action units) are shown in table 3.

\begin{tabular}{llll}
\hline AU & Description & Mean & S.D. \\
\hline \#1 & Inner Brow Raiser & 0.2241875 & 0.828964 \\
\#02 & Outer Brow Raiser & 0.8438199 & 2.983631 \\
\#04 & Brow Lowerer & 1.6331450 & 3.386449 \\
\#05 & Upper Lid Raiser & 0.0007039 & 0.014619 \\
\#06 & Cheek Raiser & 0.3656085 & 1.102261 \\
\#07 & Lid Tightener & 4.7862020 & 3.717168 \\
$\# 09$ & Nose Wrinkler & 0.3121869 & 1.123619 \\
$\# 10$ & Upper Lip Raiser & 3.0468000 & 4.136546 \\
\#12 & Lip Corner Puller & 2.1374790 & 4.339763 \\
\#14 & Dimpler & 1.1437480 & 2.370782 \\
$\# 15$ & Lip Corner Depres. & 1.1359810 & 2.868241 \\
\#17 & Chin Raiser & 0.3302074 & 1.431972 \\
\#18 & Lip Puckerer & 0.0199445 & 0.190537 \\
\#20 & Lip Stretcher & 0.6673705 & 1.884571 \\
\#23 & Lip Tightener & 2.3990960 & 4.580448 \\
\#24 & Lip Pressor & 0.7968977 & 1.718637 \\
\#25 & Lips Part & 0.5322683 & 2.178169 \\
\#26 & Jaw Drop & 0.0010564 & 0.018516 \\
\#27 & Mouth Stretch & 0.0052456 & 0.070696 \\
\#43 & Eyes Closed & 1.5039590 & 4.080349 \\
\hline
\end{tabular}

Table 3: Statistics (means and standard deviations) of facial action units.

\subsection{User identification by Random Forests}

In this study, the Random Forests (RF) method was used to identify a user based on its facial action units data. RF is a machine learning classifier which is based on an ensemble (a bag) of unpruned decision trees [21]. Ensemble methods are related to the idea that an aggregated decision from multiple experts is often superior to a decision from a single system. The classification decision is built on a majority vote principle based on all trees of the RF. The conceptual idea underlying a decision tree is to recursively identify a predictor that allows the sample to be split in two subparts that are as homogeneous as possible with regard to the classification task at hand. For binary predictors (yes/no) the split point of the variable is self-evident; for polytomous or continuous predictors the algorithms identify the most selective split point for the dependent variable using Gini impurity as a measure. In this way, a tree-like structure is built. The procedure is repeated until a stop signal is reached - e.g. all cases are classified, or the algorithm cannot improve the accuracy of the classification anymore [21]. Such types of algorithms are called recursive partitioning because the sample is subdivided (i.e. partitioned) into smaller parcels in a reiterated manner.

Since RF is unmatched in its accuracy among current machine learning algorithms, RF has been successfully ap- 
plied to a number of different neuro- and bio-science related research problems such as brain imaging [22], gene expression [23], biomarker identification [24], and information systems [25,26]. In particular, RFs are especially useful in, but not limited to, "small $n$, large $p$ " problems, where the number of predictor variables $p$ is larger than the number of cases $n$. Even with sufficiently large samples RF can be a valuable tool, as they allow the delineation of statistical properties such as non-linear trends, high-degree interaction, and correlated predictors. Additionally, assumptions that are needed for classical multivariate analyses such as homoscedasticity (homogeneity of variance), linear associations between variables, or metric variable levels are not necessary [21].

\section{Results}

For RF training and analyses we applied the randomForest v4.6-12 package within a R x64 3.4.0 environment [27] running on a 32 GB RAM Lenovo W530 workstation.

For training and evaluation of the Random Forests decision tree we split the $n=1,413$ sample in a training partition $\left(\mathrm{n}_{\mathrm{T}}=1,109\right)$ and an evaluation partition $\left(\mathrm{n}_{\mathrm{E}}=304\right)$. trees.

The Random Forests classifier was built using 612 voting

\subsection{Performance evaluation}

We evaluated the developed user identifier in terms of class-averaged sensitivity (true positive rate), specificity (true negative rate), precision (positive predictive value), negative predictive value, and balanced accuracy. As shown in table 4 the classifier achieved excellent performance values.

\begin{tabular}{ll}
\hline Performance indicator & Value \\
\hline True positive rate & 0.945544554 \\
True negative rate & 0.999221405 \\
Positive predictive value & 0.924092409 \\
Negative predictive value & 0.999210552 \\
Prevalence & 0.009803922 \\
Balanced accuracy & 0.972395410 \\
\hline
\end{tabular}

Table 4: Evaluation indicators of the Random Forests classifier.

The results indicate the trained classifier has a balanced prediction accuracy of 97.24 percent - just based on facial expressions. The calculated multi-class area under the curve is 0.9659 .

In order to prevent unauthorized access by means of secure access control systems the specificity (true negative rate) is an important indicator. Based on facial expressions alone, the trained classifier shows a specificity of 99.92 percent. When the facial expressions-based classifier is combined with a PIN request - which is a rule in access validation systems $[2,3]$ - we reached a very good false positive rate of only $7.633284 \mathrm{e}-06$ (over 99.999 percent specificity).

\subsection{Importance of specific facial action units}

The trained Random Forests classifier can be analyzed to investigate the importance of specific facial action units. The Random Forests classifier uses Gini impurity as the measure of choice to split a sub-tree. Gini impurity is calculated by $1-\sum_{i=1}^{C} p_{i}^{2}$, where $p_{i}$ is the proportion of instances in the dataset that take the $i$ th value of the target attribute and $C$ is the number of classes.

Table 5 shows the total decrease in Gini impurities from splitting on the variable, averaged over all trees.

\begin{tabular}{llr}
\hline AU & Description & Decrease of Gini impurity \\
\hline$\# 01$ & Inner Brow Raiser & 28.4668273 \\
$\# 02$ & Outer Brow Raiser & 40.8244234 \\
$\# 04$ & Brow Lowerer & 47.7284057 \\
$\# 05$ & Upper Lid Raiser & 0.5674926 \\
$\# 06$ & Cheek Raiser & 27.8330493 \\
$\# 07$ & Lid Tightener & 102.0712682 \\
$\# 09$ & Nose Wrinkler & 32.7430243 \\
$\# 10$ & Upper Lip Raiser & 77.4078558 \\
$\# 12$ & Lip Corner Puller & 55.2664149 \\
$\# 14$ & Dimpler & 58.5006261 \\
$\# 15$ & Lip Corner Depres. & 56.2202643 \\
$\# 17$ & Chin Raiser & 18.8375354 \\
$\# 18$ & Lip Puckerer & 4.7201763 \\
$\# 20$ & Lip Stretcher & 39.0406277 \\
$\# 23$ & Lip Tightener & 65.2249056 \\
$\# 24$ & Lip Pressor & 57.5426887 \\
$\# 25$ & Lips Part & 20.7227229 \\
$\# 26$ & Jaw Drop & 0.2332505 \\
$\# 27$ & Mouth Stretch & 1.9060906 \\
$\# 43$ & Eyes Closed & 45.8510198 \\
\hline
\end{tabular}

Table 5: Variable importance measured by mean decrease of Gini impurity.

As shown in table 5 the Lid Tightener (action unit 7), Upper Lip Raiser (action unit 10) and Lip Tightener (action unit 23) are the three most important variables to identify a person (unaffected by its emotions). Figure 9 highlights those facial muscles which are most important for robust user identification (orbicularis oculi, pars palpebralis; levator labii superioris; orbicularis oris).

\section{Discussion}

User identification methods should be evaluated by its performance, acceptability, and circumvention [4].

(1) Performance: As demonstrated in table 4 the user identification method performed very well. With a balanced accuracy of 97.24 percent just based on facial action unit analysis and a true negative rate of 99.92 percent it 


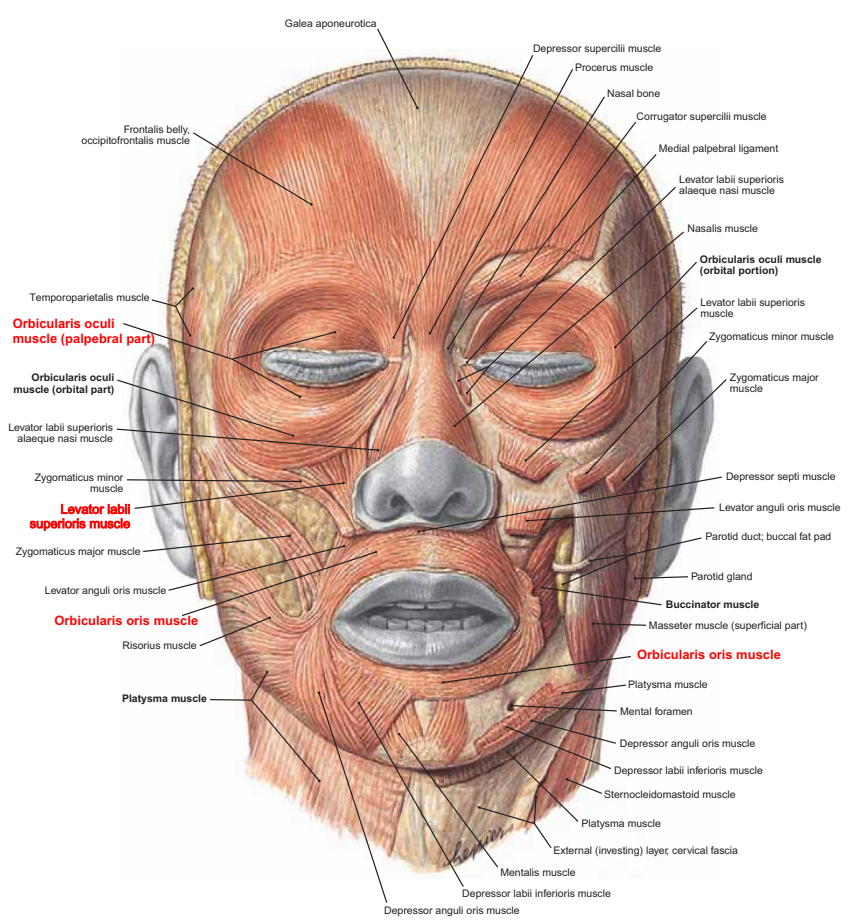

Figure 9: Most important facial muscles for user identification. Adapted from [12, p. 580].

outperforms non-contactless approaches such as electroencephalography (from 72 percent accuracy using single eventrelated potentials to 96.7 percent accuracy using multiple epochs [28]). It was further shown that the proposed user identification method is robust against the user's emotions. In combination with a PIN request the specificity rises to over 99.999 percent.

To compare our method to other available biometric technologies, we summarize technology-specific false positive rates in table 6 .

\begin{tabular}{ll}
\hline Biometric technology & False positive rate \\
\hline Keystrokes & 0.07 \\
Fingerprint & 0.02 \\
Hand geometry & 0.02 \\
Voice & 0.02 \\
Static face & 0.01 \\
Iris & 0.0094 \\
\hline
\end{tabular}

Our method (facial action units) $\quad 0.0000076$

Table 6: False positive rates of available technologies from the biometric authentication review [29] in comparison with our method.

(2) Acceptability: In addition, the acceptability of the camera-based proposed identification method is high since it is easy to use, cost efficient, non-invasive and contactfree, whereas other physiological/biometric data from electrodermal activity, heart-rate, electroencephalography, fa- cial electromyography, functional near infrared spectroscopy cannot be captured by contact-free methods, and functional magnetic resonance imaging, positron emission tomography or magnetoencephalography are very cost-expensive [30,31].

(3) Circumvention: While photography-based approaches (iris, retinal, and face scans) can be tricked by using previously captured images [2]-[4], the method proposed here uses living data from facial muscles.

\subsection{Theoretical implications}

Our research offers insights into those facial muscles which are most suitable for user identification. The three most important facial muscles for robust user identification are the orbicularis oculi muscle (pars palpebralis), levator labii superioris muscle, and orbicularis oris muscle.

\subsection{Practical implications}

A precondition of "the next generation of humancomputer interfaces [...] able to facilitate more lifelike and natural interactions with humans" [32, p.62] is the automatic identification of the interacting human user through the machine. The camera-based approach proposed here allows such an automatic user identification. While the approach is easy to use, cost efficient, and contact-free, it is suitable for applications in human-machine interaction settings. Furthermore, the identification method can also be used in human-machine interaction with digital avatars [1].

Because of the very high true negative rate of 99.92 percent and over 99.999 percent in combination with a PIN request the method is applicable for access control systems.

\section{Conclusion}

We built a Random Forests classifier identifying a user based on its facial action unit activity that is captured by an ordinary webcam. The performance evaluation revealed a higher accuracy and - what is important for secure access systems - a higher true negative rate in comparison to existing approaches. In combination with a PIN request the specificity (true negative rate) rises to over 99.999 percent. Because our method uses living data (facial action unit activity related to facial muscle activity) from humans it is hard to circumvent the approach. In addition, the proposed method is also easy to use, cost efficient, non-invasive and contact-free.

As demonstrated in table 6, with a false positive rate of of only 7.633284e-06 (over 99.999 percent specificity) our method outperforms all other currently available biometric technologies.

\subsection{Limitations}

While our user identification method is useful for practical applications, a straight head orientation of the user in front of the camera is important to properly detect the facial 
action units. Another limitation is related to the fact that the facial action units of every participant were recorded in a small time frame (about 8 minutes per participant). In order to address the concern of the small time frame we afterwards analyzed data from another experiment [33] and picked those nine participants where we captured more than one hour of facial action data. On the basis of this facial action data we trained and re-evaluated our Random Forests algorithm. As a result, we can report that we were able to robustly identify each participant correctly in all smaller time frames during the whole experiment (accuracy of 100 percent). However, the sample size of this retest is small and we captured the facial action data only on one day for each participant. That is why our future research will use a test / re-test experimental setup to train and evaluate our classifier at one meeting and evaluate the classifier again a few days later $[34,35]$.

\subsection{Future research}

Beyond relaxing the limitations mentioned before, future research should focus on the possibility of combining biometric authentication methods [36].

\section{Acknowledgments}

We would like to thank the HICSS-51 reviewers and the minitrack chair, who each provided very helpful comments on the refinement of the paper.

\section{References}

[1] M. Seymour, K. Riemer, and J. Kay, "Interactive Realistic Digital Avatars - Revisiting the Uncanny Valley," in HICSS-50 Proc., 2017, pp. $547-556$.

[2] S. Boukhonine, V. Krotov, and B. Rupert, "Future Security Approaches and Biometrics," Communications of the Association for Information Systems, vol. 16, pp. 937-966, 2005.

[3] I. Martinovic, K. B. Rasmussen, M. Roeschlin, and G. Tsudik, "PulseResponse: Exploring Human Body Impedance for Biometric Recognition," ACM Transactions on Privacy and Security (TOPS), vol. 20, no. 2, p. Article No. 6, 2017.

[4] A. Jain, L. Hong, and S. Pankanti, "Biometric Identification," Communications of the ACM, vol. 43, no. 2, pp. 90-98, 2000.

[5] L. Benedikt, D. Cosker, P. L. Rosin, and D. Marshall, "Assessing the Uniqueness and Permanence of Facial Actions for Use in Biometric Applications," IEEE Transactions on Systems, Man, and Cybernetics - Part A: Systems and Humans, vol. 40, no. 3, pp. 449-460, 2010.

[6] P. Ekman, W. V. Friesen, and J. C. Hager, The Facial Action Coding System, 2nd ed. London: Weidenfeld \& Nicolson, 2002.

[7] M. Hibbeln, J. L. Jenkins, C. Schneider, J. S. Valacich, and M. Weinmann, "How Is Your User Feeling? Inferring Emotion Through Human-Computer Interaction Devices," MIS Quarterly, vol. 41, no. 1, pp. 1-21, 2016.

[8] P. J. Lang, M. M. Bradley, and B. N. Cuthbert, "International affective picture System (IAPS): Affective ratings of pictures and instruction manual," Univ. of Florida, Tech. Rep., 2008.

[9] N. Kock, "Information Systems Theorizing Based on Evolutionary Psychology: An Interdisciplinary Review and Theory Integration Framework," MIS Quarterly, vol. 33, no. 2, pp. 395-418, 2009.

[10] S. Djamasbi, M. Siegel, and T. S. Tullis, "Faces and Viewing Behavior: An Exploratory Investigation," AIS Transactions on Human-Computer Interaction, vol. 4, no. 3, pp. 190-211, 2012.
[11] S. Brown, R. Fuller, and S. M. Thatcher, "Impression Formation and Durability in Mediated Communication," Journal of the Association for Information Systems, vol. 17, no. 9, pp. 614-647, 2016.

[12] C. D. Clemente, Anatomy: A Regional Atlas of the Human Body, 6th ed. Baltimore, MD, US: Wolters Kluwer, 2011.

[13] P. Ekman and W. V. Friesen, Facial Action Coding System: A Technique for the Measurement of Facial Movement. Palo Alto, CA: Consulting Psychologists Press, 1978.

[14] Y.-1. Tian, T. Kanade, and J. F. Cohn, "Recognizing Action Units for Facial Expression Analysis," IEEE Transactions on Pattern Analysis and Machine Intelligence, vol. 23, no. 2, pp. 97-115, 2001.

[15] P. Ekman, "Facial Expression and Emotion," American Psychologist, vol. 48, no. 4, pp. 384-392, 1992.

[16] P. Lucey, J. F. Cohn, T. Kanade, J. Saragih, Z. Ambadar, and I. Matthews, "The Extended Cohn-Kanade Dataset (CK+): A complete dataset for action unit and emotion-specified expression," in 2010 IEEE Computer Vision and Pattern Recognition Workshops (CVPRW) Proceedings, 2010.

[17] J. vom Brocke and T.-P. Liang, "Guidelines for Neuroscience Studies in Information Systems Research," Journal of Management Information Systems, vol. 30, no. 4, pp. 211-234, 2014.

[18] R. E. Kirk, Experimental Design: Procedures for the Behavioral Sciences, 4th ed. Sage, 2013

[19] K. Takeo, J. F. Cohn, and Y. Tian, "Comprehensive Database for Facial Expression Analysis," in Proc. of 4th IEEE Int. Conference on Automatic Face and Gesture Recognition, 2000.

[20] J. F. Cohn, Z. Ambadar, and P. Ekman, Observer-Based Measurement of Facial Expression With the Facial Action Coding System, ser. Series in Affective Science. New York, NY, US: Oxford University Press, 2007, ch. 13, pp. 203-221, in Coan, J. A.; Allen, J. J. B. (Eds): Handbook of Emotion Elicitation and Assessment.

[21] L. Breiman, "Random Forests," Machine Learning, vol. 45, no. 1, pp. 5-32, 2001.

[22] K. Kačar, M. A. Rocca, M. Copetti, S. Sala, v. Mesaroš, T. Stosić Opinćal, D. Caputo, M. Absinta, J. Drulović, V. S. Kostić, G. Comi, and M. Filippi, "Overcoming the ClinicalMR Imaging Paradox of Multiple Sclerosis: MR Imaging Data Assessed with a Random Forest Approach," American Journal of Neuroradiology, vol. 32, no. 11, pp. 2098-2102, 2011.

[23] R. Díaz-Uriarte and S. A. de Andrés, "Gene selection and classification of microarray data using random forest," BMC Bioinformatics, vol. 7 , no. 3, 2006.

[24] J. Zhang, I. Sokal, E. R. Peskind, J. F. Quinn, J. Jankovic, C. Kenney, K. A. Chung, S. P. Millard, J. G. Nutt, and T. J. Montine, "CSF Multianalyte Profile Distinguishes Alzheimer and Parkinson Diseases," American Journal of Clinical Pathology, vol. 129, no. 4, pp. 526-529, 2008.

[25] J. Ali, R. Khan, N. Ahmad, and I. Maqsood, "Random Forests and Decision Trees," International Journal of Computer Science Issues, vol. 9, no. 5, pp. 272-278, 2012.

[26] R. Buettner, S. Sauer, C. Maier, and A. Eckhardt, "Towards ex ante Prediction of User Performance: A novel NeuroIS Methodology based on Real-Time Measurement of Mental Effort," in HICSS-48 Proc. 2015, pp. 533-542.

[27] R Core Team, R: A Language and Environment for Statistical Computing, R Foundation for Statistical Computing, Vienna, Austria, 2017.

[28] T. Koike-Akino, R. Mahajan, T. K. Marks, Y. Wang, S. Watanabe, O. Tuzel, and P. Orlik, "High-Accuracy User Identification Using EEG Biometrics," in 38th IEEE Annual International Conference of the Engineering in Medicine and Biology Society (EMBC), 2016.

[29] D. Bhattacharyya, R. Ranjan, F. Alisherov, and M. Choi, "Biometric Authentication: A Review," International Journal of $u$ - and e-Service, Science and Technology, vol. 2, no. 3, pp. 13-28, 2009.

[30] A. Dimoka, R. D. Banker, I. Benbasat, F. D. Davis, A. R. Dennis, D. Gefen, A. Gupta, A. Ischebeck, P. H. Kenning, P. A. Pavlou, G. Müller-Putz, R. Riedl, J. vom Brocke, and B. Weber, "On the Use of Neurophysiological Tools in IS Research: Developing a Research Agenda for NeuroIS," MIS Quarterly, vol. 36, no. 3, pp. 679-A19, 2012.

[31] D. Gefen, H. Ayaz, and B. Onaral, "Apply Functional Near Infrared (fNIR) Spectroscopy to Enhance MIS Research," AIS Transactions on 
Human-Computer Interaction, vol. 6, no. 3, pp. 55-73, 2014.

[32] D. C. Derrick, J. L. Jenkins, and J. F. Nunamaker, Jr., "Design Principles for Special Purpose, Embodied, Conversational Intelligence with Environmental Sensors (SPECIES) Agents," AIS Transactions on Human-Computer Interaction, vol. 3, no. 2, pp. 62-81, 2011.

[33] R. Buettner, "Asking both the Users Brain and its Owner using Subjective and Objective Psychophysiological NeuroIS Instruments," in ICIS 2017 Proceedings, 2017.

[34] P. M. Podsakoff, S. B. MacKenzie, J.-Y. Lee, and N. P. Podsakoff, "Common Method Biases in Behavioral Research: A Critical Review of the Literature and Recommended Remedies," Journal of Applied Psychology, vol. 88, no. 5, pp. 879-903, 2003.

[35] P. M. Podsakoff, S. B. MacKenzie, and N. P. Podsakoff, "Sources of Method Bias in Social Science Research and Recommendations on How to Control It," Annual Review of Psychology, vol. 63, pp. 539-69, 2012.

[36] R. Snelick, U. Uludag, A. Mink, M. Indovina, and A. Jain, "LargeScale Evaluation of Multimodal Biometric Authentication Using State-of-the-Art Systems," IEEE Transactions on Pattern Analysis and Machine Intelligence, vol. 27, no. 3, pp. 450-455, 2005.

\section{Appendix}

In the following we report the perceptive ratings of the remaining IAPS stimuli used in the experiment.

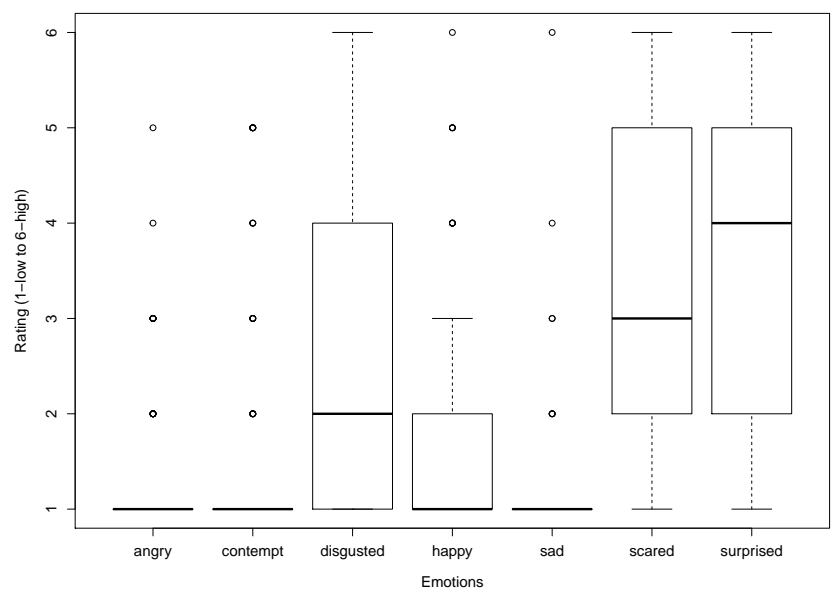

Figure 10: Perceptive rating of snake stimulus (\#1050).

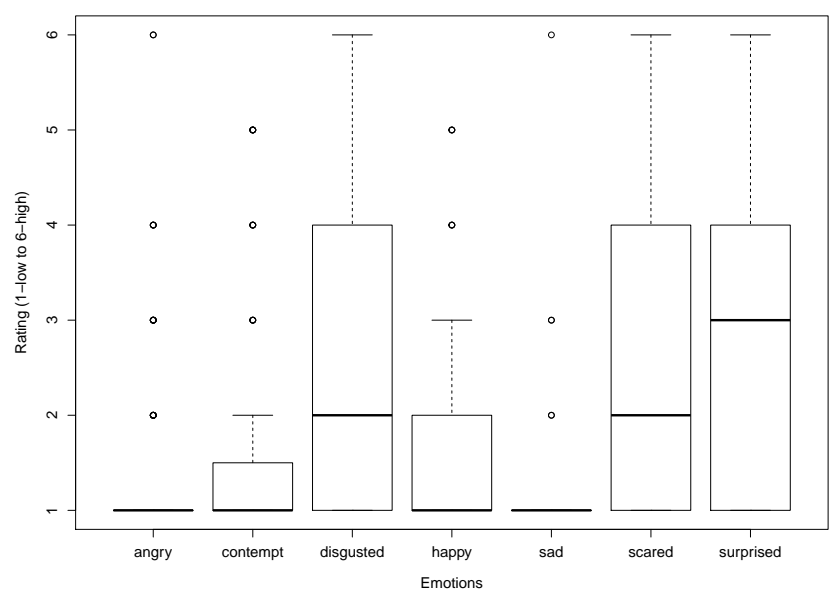

Figure 11: Perceptive rating of spider stimulus (\#1201).

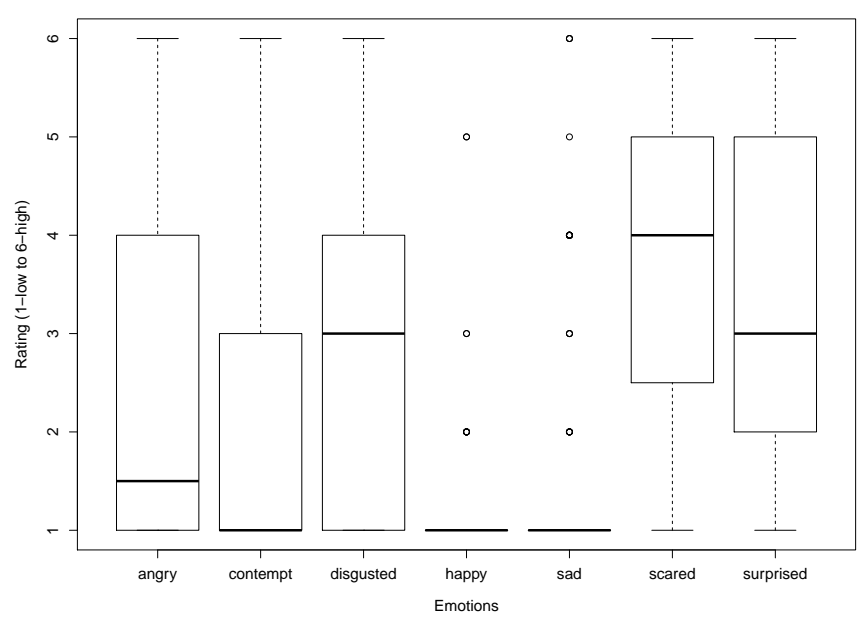

Figure 12: Perceptive rating of pit bull stimulus (\#1300).

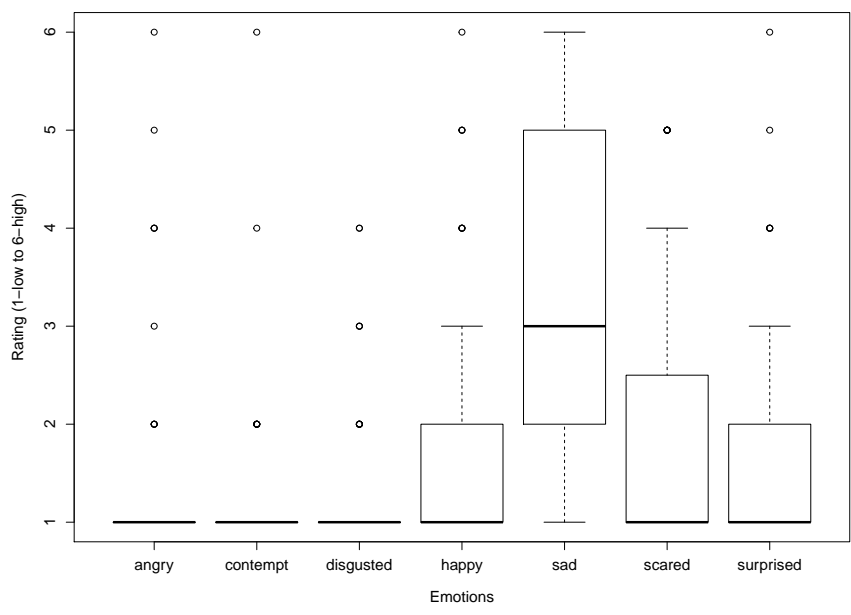

Figure 13: Perceptive rating of cemetery stimulus (\#9001).

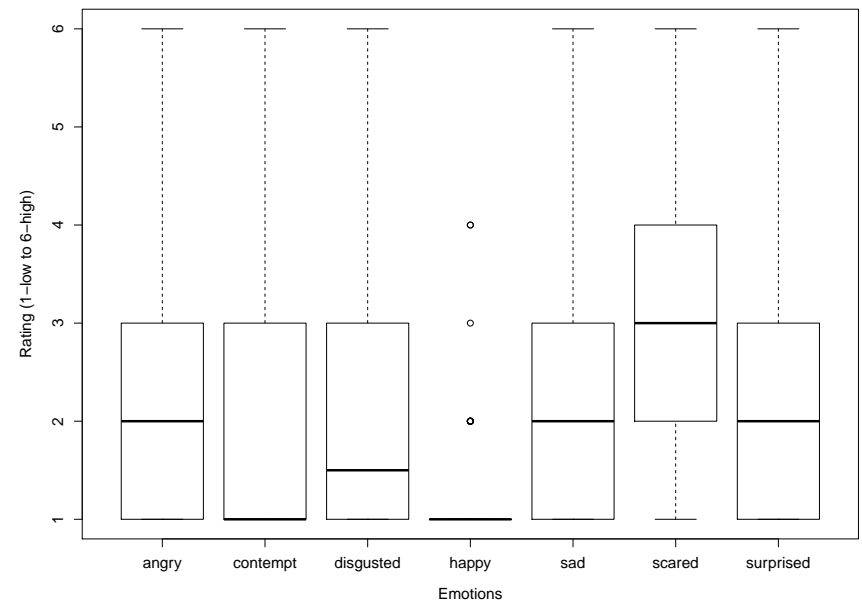

Figure 14: Perceptive rating of toxic waste stimulus (\#9270). 


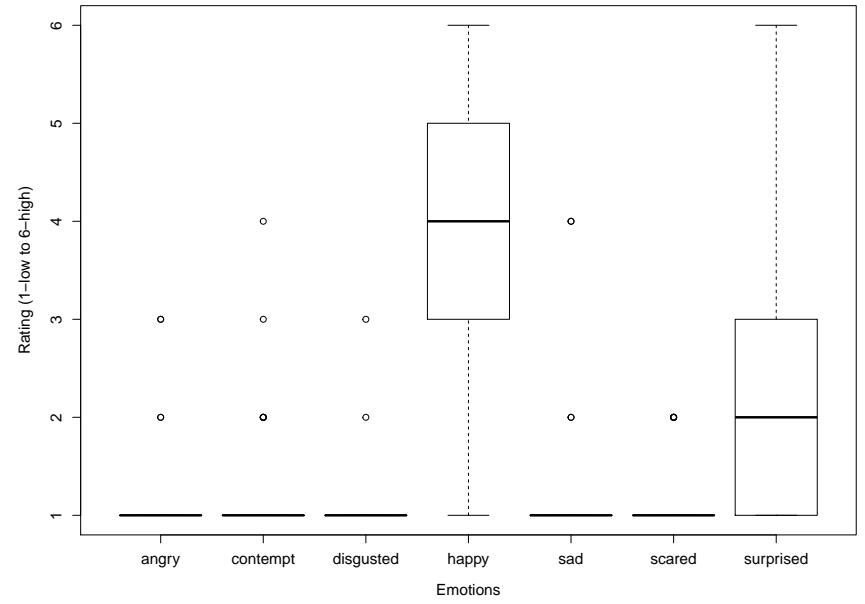

Figure 15: Perceptive rating of woman stimulus (\#2030).

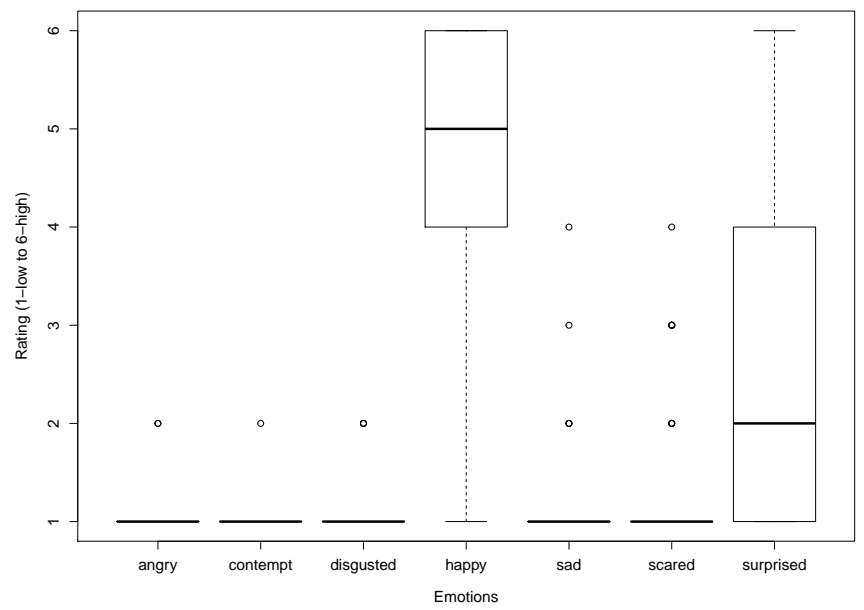

Figure 16: Perceptive rating of boy stimulus (\#2306).

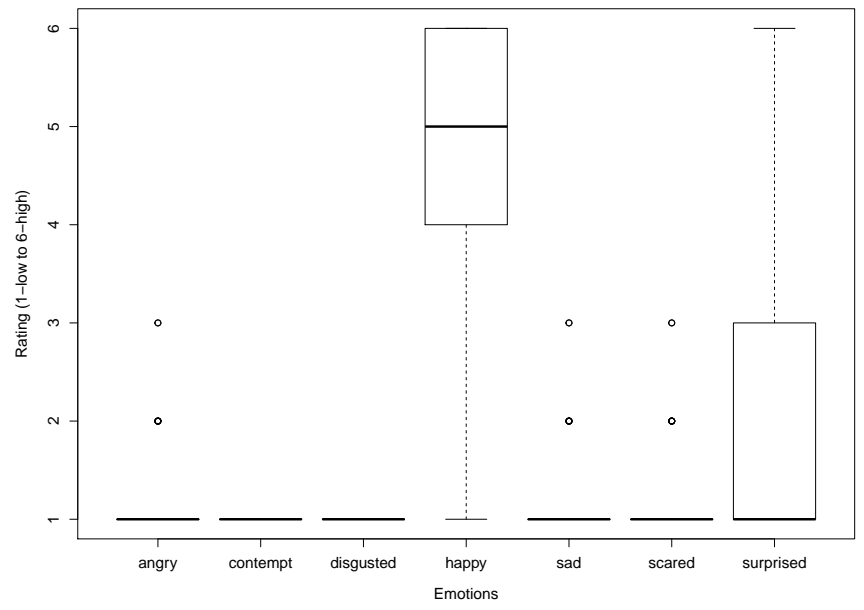

Figure 17: Perceptive rating of mother stimulus (\#2311).

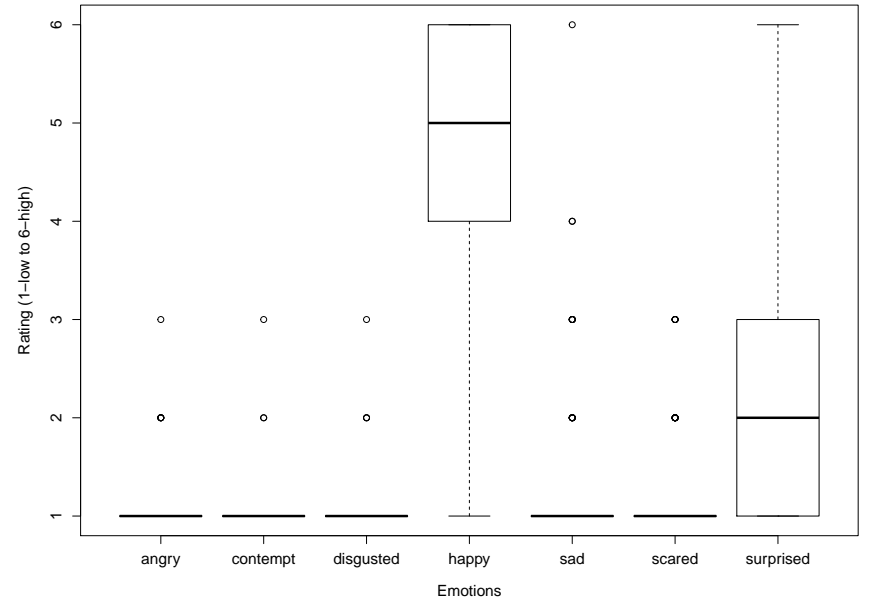

Figure 18: Perceptive rating of children stimulus (\#2341).

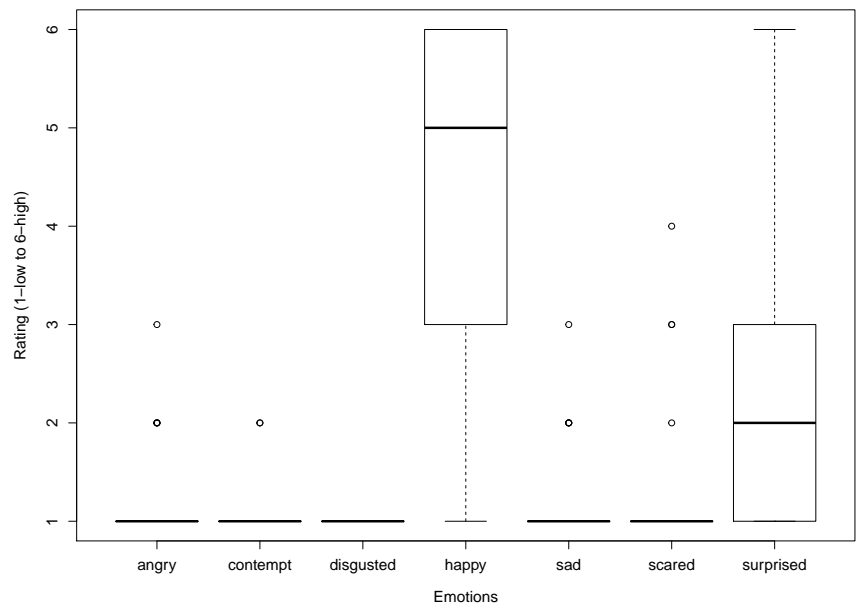

Figure 19: Perceptive rating of athletes stimulus (\#8540).

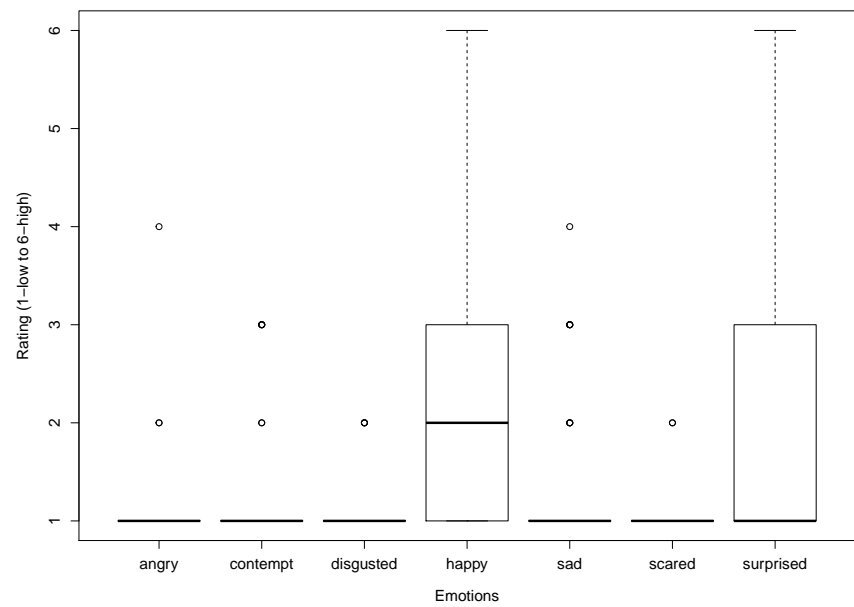

Figure 20: Perceptive rating of mug stimulus (\#7009). 